\title{
A new flexible Weibull distribution
}

\author{
Sangun Park ${ }^{a}$, Jihwan Park ${ }^{1, a}$, Youngsik Choi $^{a}$ \\ ${ }^{a}$ Department of Applied Statistics, Yonsei University, Korea
}

\begin{abstract}
Many of studies have suggested the modifications on Weibull distribution to model the non-monotone hazards. In this paper, we combine two cumulative hazard functions and propose a new modified Weibull distribution function. The newly suggested distribution will be named as a new flexible Weibull distribution. Corresponding hazard function of the proposed distribution shows flexible (monotone or non-monotone) shapes. We study the characteristics of the proposed distribution that includes ageing behavior, moment, and order statistic. We also discuss an estimation method for its parameters. The performance of the proposed distribution is compared with existing modified Weibull distributions using various types of hazard functions. We also use real data example to illustrate the efficiency of the proposed distribution.
\end{abstract}

Keywords: Weibull distribution, modified Weibull distribution, bathtub shape, hazard function, maximum likelihood estimate, reliability

\section{Introduction}

Weibull distribution has been widely used in various fields such as reliability engineering due to its flexibility in fitting failure times, where its survival function is given as

$$
\bar{F}(t)=\exp \left(-(\theta t)^{\lambda}\right), \quad t>0,
$$

with parameters $\lambda, \theta>0$.

The corresponding hazard function (failure rate function) can then be written as

$$
h(t)=\lambda \theta^{\lambda} t^{\lambda-1} .
$$

However, Weibull distribution is inappropriate to model the non-monotone hazard rate such as bathtubshaped hazard rate because Weibull distribution can produce only monotonic hazard rates. Hence, many of modifications of Weibull distribution have been suggested, which can fit the non-monotone hazard rate.

It may be a natural approach to combine two different survival functions (with increasing and decreasing hazards) and generate a distribution function as

$$
\bar{F}(t)=\alpha \bar{F}_{1}(t)+(1-\alpha) \bar{F}_{2}(t),
$$

where $0<\alpha<1$, which is well-known as a mixture of distributions, or

$$
\bar{F}(t)=\bar{F}_{1}^{\alpha}(t) \times \bar{F}_{2}^{\beta}(t),
$$

\footnotetext{
${ }^{1}$ Corresponding author: Department of Applied Statistics, Yonsei University, 50, Yonsei-ro, Seodaemun-gu, Seoul 03722, Korea. E-mail: jh.park@yonsei.ac.kr
}

Published 30 September 2016 / journal homepage: http://csam.or.kr (c) 2016 The Korean Statistical Society, and Korean International Statistical Society. All rights reserved. 
with parameters $\alpha, \beta>0$.

The cumulative distribution function can be written in terms of the cumulative hazard function $H(x)$ as

$$
F(t)=1-e^{-H(t)},
$$

where the cumulative hazard function satisfies the following properties as

1. $H(t)$ is nonnegative and increasing,

2. $\lim _{t \rightarrow 0} H(t)=0$ and $\lim _{t \rightarrow \infty} H(t)=\infty$.

Hence, we can also combine two cumulative hazard functions to generate a distribution function as

$$
H(t)=\alpha H_{1}(t)+\beta H_{2}(t),
$$

which is eventually equivalent to (1.1), or

$$
H(t)=H_{1}^{\alpha}(t) \times H_{2}^{\beta}(t),
$$

with parameters $\alpha, \beta>0$. We can introduce further an intercept parameter in (1.3) to add more flexibility.

The modified Weibull distributions suggested by Xie and Lai (1995), Almalki and Yuan (2013), and Lemonte et al. (2014) belong to the class (1.2), and the modified Weibull distributions suggested by Xie et al. (2002), Lai et al. (2003), Nadarajah and Kotz (2005), Bebbington et al. (2007) and Aryal and Elbatal (2015) belong to the class (1.3), and Park and Park (2016) discussed its generalization. Only the difference in past works is the choice of cumulative hazard functions. If we combine different types of hazard functions, where one is increasing and the other is decreasing, the hazard functions of the above distribution functions are expected to be flexible. However, the popular choices in past works include $\exp (t)$ and $\log (t)$, which are not appropriate as cumulative hazard functions.

In this paper, we choose $\exp (t)-1$ and $\log (t+1)$ as two cumulative hazard functions and produce a new flexible Weibull distribution (NFW) by following the aforementioned approach (1.3) as

$$
\log H_{N F W}(t)=\mu+\alpha \log (\exp (t)-1)+\beta \log (\log (t+1)) .
$$

We provide the properties including characterization of hazard function and ageing behavior. The proposed distribution is also compared with some current modified Weibull distributions for various types of distribution functions and real data example.

\section{New flexible Weibull distribution and its properties}

We propose a new modified Weibull distribution as

$$
\log H_{N F W}(t ; \mu, \alpha, \beta)=\mu+\alpha \log (\exp (t)-1)+\beta \log (\log (t+1)),
$$

which we call new flexible Weibull distribution.

Since both $\exp (t)-1$ and $\log (t+1)$ satisfy the properties of the cumulative hazard function, the resulting new flexible Weibull distribution becomes theoretically rigorous and more flexible compared to other modified Weibull distributions. The hazard function corresponding to $\alpha \log (\exp (t)-1)$ shows 
the bathtub-shape $(\alpha<1)$ or the increasing shape $(\alpha \geq 1)$, whereas the hazard function corresponding to $\beta \log (\log (t+1))$ may be decreasing $(\beta \leq 1)$ or upside-down bathtub-shaped $(\beta>1)$.

The cumulative hazard function of the new flexible Weibull distribution is given by

$$
H_{N F W}(t ; \mu, \alpha, \beta)=\exp (\mu)\{\exp (t)-1\}^{\alpha}\{\log (t+1)\}^{\beta},
$$

and the corresponding hazard function has the following form

$$
\begin{aligned}
h_{N F W}(t ; \mu, \alpha, \beta)= & \exp (\mu)\{\exp (t)-1\}^{\alpha-1}\{\log (t+1)\}^{\beta-1} \\
& \times\left[\alpha \exp (t) \log (t+1)+\{\exp (t)-1\} \frac{\beta}{t+1}\right] .
\end{aligned}
$$

The cumulative distribution function of the new flexible Weibull distribution can be written as

$$
F_{N F W}(t ; \mu, \alpha, \beta)=1-\exp \left[-\exp (\mu)\{\exp (t)-1\}^{\alpha}\{\log (t+1)\}^{\beta}\right],
$$

and its probability density function can be obtained as

$$
\begin{aligned}
f_{N F W}(t ; \mu, \alpha, \beta)= & \exp (\mu)\{\exp (t)-1\}^{\alpha-1}\{\log (t+1)\}^{\beta-1} \\
& \times\left[\alpha \exp (t) \log (t+1)+\{\exp (t)-1\} \frac{\beta}{t+1}\right] \\
& \times \exp \left[-\exp (\mu)\{\exp (t)-1\}^{\alpha}\{\log (t+1)\}^{\beta}\right] .
\end{aligned}
$$

Figures 1 and 2 shapes of hazard function $h_{N F W}(t)$ relating to the change of $\alpha$ and $\beta$, with $\mu$ fixed to 0 . As shown in both figures, the hazard functions of the new flexible Weibull distribution can cover increasing, decreasing, bathtub-shaped, modified bathtub-shaped and upside-down bathtub-shaped failure rates.

We also study the limiting behavior of the hazard function of the hazard function. Note that $\lim _{t \rightarrow \infty} h_{N F W}(t ; \mu, \alpha, \beta)=0$ when $\alpha$ is close to 0 and $h_{N F W}(t)$ goes to $\infty$ otherwise. It is straightforward that $\lim _{t \rightarrow 0} h_{N F W}(t ; \mu, \alpha, \beta)=0$ when at least one of the parameters $\alpha$ or $\beta$ is greater or equal to 1. However when neither $\alpha$ nor $\beta$ is greater or equal to 1 , the limiting behavior varies as follows, depending on the parameters. Figure 2 illustrates the different behaviors of hazard function as $t$ goes to 0 , by an example of changing $\beta$ when $\alpha$ is fixed.

1. If $\alpha+\beta$ is less than 1 ,

$$
\lim _{t \rightarrow 0} h_{N F W}(t ; \mu, \alpha, \beta)=\infty .
$$

2. If $\alpha+\beta$ is equal to 1 ,

$$
\lim _{t \rightarrow 0} h_{N F W}(t ; \mu, \alpha, \beta)=\exp (\mu) .
$$

3. If $\alpha+\beta$ is greater than 1 ,

$$
\lim _{t \rightarrow 0} h_{N F W}(t ; \mu, \alpha, \beta)=0 .
$$

Limiting behavior plays an important role on the shape of the hazard function. The resulting hazard function is only decreasing if a limiting behavior is fixed, for example, to be $h(0)=\infty$ and 



Figure 1: Hazard function of new flexible Weibull distribution with $\mu=0$.

Hazard : Alpha $=0.2$

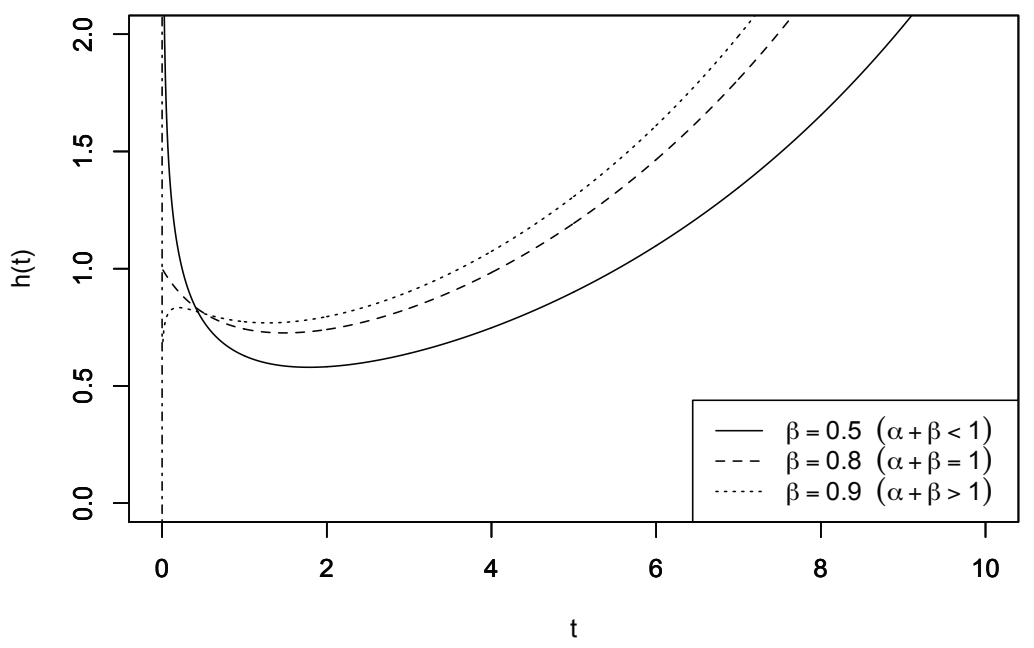

Figure 2: Change of limiting behavior of hazard function when $\alpha=0.2$. 

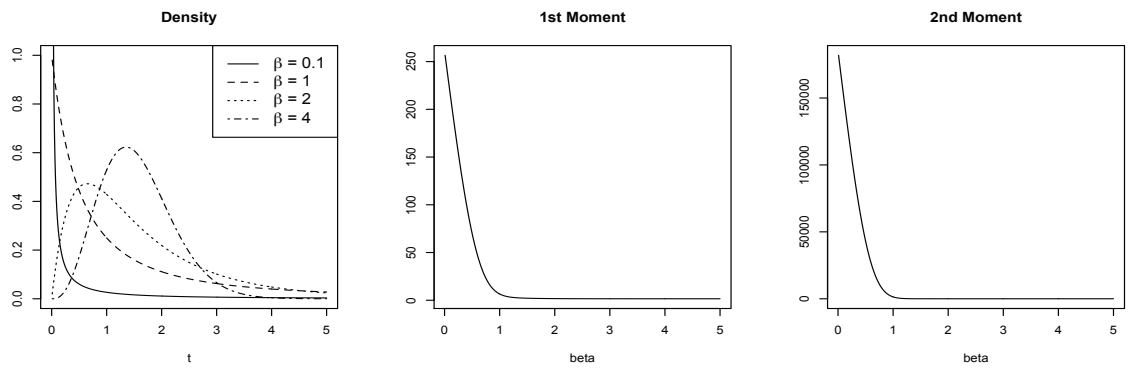

Figure 3: Change of density and moment when $\alpha=1 e-06$.
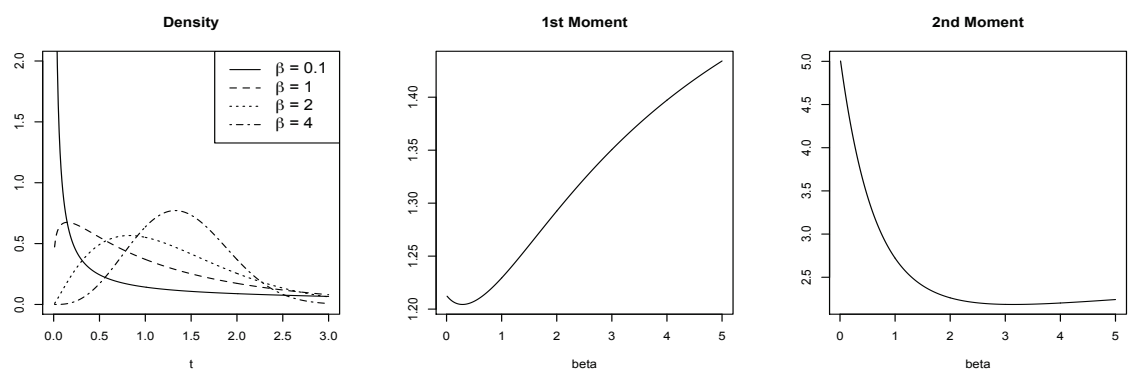

Figure 4: Change of density and moment when $\alpha=0.2$.
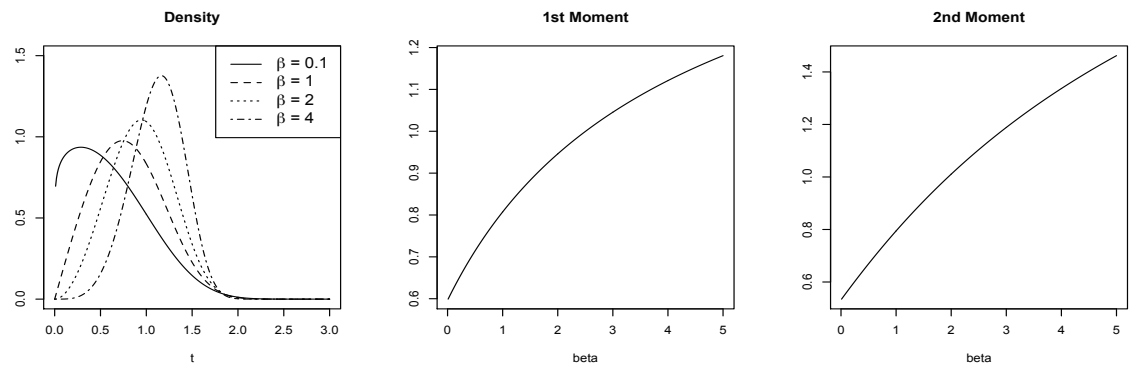

Figure 5: Change of density and moment when $\alpha=1$.

$h(\infty)=0$. Most previous studies have fixed limiting behaviors, which result in limited types of capable hazard functions. The new flexible Weibull distribution, however, is able to cover various types of hazard functions by an appropriate choice of parameters.

We can write the $r^{\text {th }}$ moment of the new flexible Weibull distribution as

$$
\begin{aligned}
E\left[T^{r}\right] & =\int_{0}^{\infty} t^{r} f(t) d t=\int_{0}^{\infty} r t^{r-1} S(t) d t \\
& =\int_{0}^{\infty} r t^{r-1} \exp \left[-\exp (\mu)\{\exp (t)-1\}^{\alpha}\{\log (t+1)\}^{\beta}\right] d t .
\end{aligned}
$$

We use Gauss-Kronrod quadrature for numerical integration since the above integral cannot be computed in closed-form. Figures 3-5 shows the changing density and moments as $\beta$ varies when $\alpha$ is fixed. 
Density : Alpha $=0.5$, Beta $=0.5$

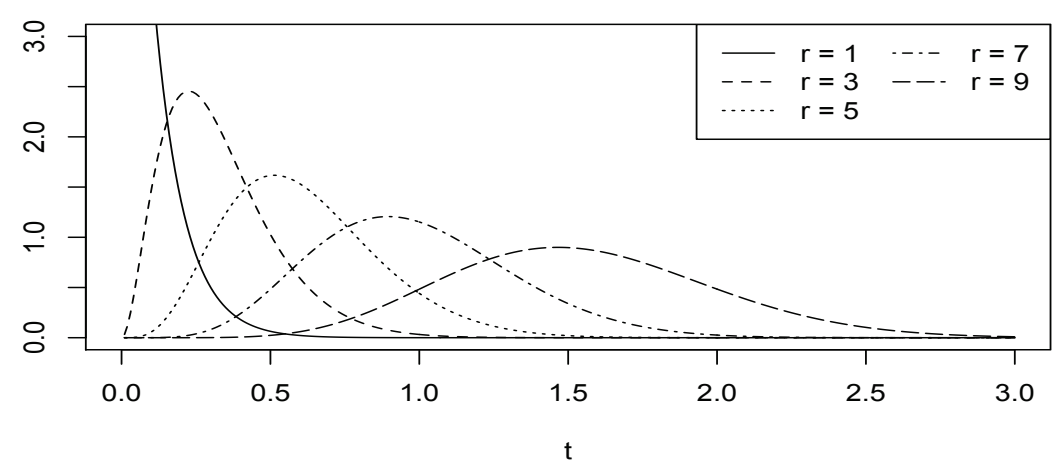

Figure 6: Probability density function of $r^{\text {th }}$ order statistic.

We also study the order statistic of the proposed distribution. We denote the probability density function of $r^{\text {th }}$ order statistic $T_{(r)}$ as $f_{r: n}(t)$. The probability density function $f_{r: n}(t)$ can be written as

$$
\begin{aligned}
f_{r: n}(t) & =\frac{1}{B(r, n-r+1)} F^{r-1}(t)[1-F(t)]^{n-r} f(t) \\
& =\frac{1}{B(r, n-r+1)} \sum_{k=0}^{r-1}\left(\begin{array}{c}
r-1 \\
k
\end{array}\right)(-1)^{k} \exp \{-H(t)(n+k+1-r)\} h(t) .
\end{aligned}
$$

Since the probability density function $f(t)$ can be written in terms of hazard function $h(t)$ and cumulative hazard function $H(t)$ as

$$
f(t)=h(x) e^{-H(t)}
$$

we can derive $f_{r: n}(t)$ as

$$
f_{r: n}(t)=\frac{1}{B(r, n-r+1)} \sum_{k=0}^{r-1}\left(\begin{array}{c}
r-1 \\
k
\end{array}\right) \frac{(-1)^{k}}{(n+k+1-r)} f\left(t ; \mu^{*}, \alpha, \beta\right),
$$

where $\mu^{*}=\mu+\log (n+k+1-r)$. Figure 6 shows the probability density function of $r^{\text {th }}$ order statistic when $\alpha$ and $\beta$ are both fixed to 0.5 and $n$ fixed to 10 .

\section{Parameter estimation}

In order to estimate the unknown parameters of the distribution, we can consider the Weibull-type probability plot employed in Bebbington et al. (2007), Lai et al. (2003) and Park and Park (2016) by letting the theoretical cumulative hazard function be as close to the empirical cumulative hazard function as

$$
\log \hat{H}(t)=\mu+\alpha \log (\exp (t)-1)+\beta \log (\log (t+1))
$$

where $\hat{H}(t)$ is the nonparametric estimate of the cumulative hazard function at $t$. 
Hence, we can obtain the (weighted) least square estimation by estimating $H(t)$ with the NelsonAalen estimator or Kaplan-Meier estimator. For simplicity, one may consider the ordinary least square estimation; however, we consider the maximum likelihood estimation as follows.

The likelihood function of the new flexible Weibull distribution given $t_{1}, \ldots, t_{n}$ has the following form as

$$
\begin{aligned}
L_{N F W}(\mu, \alpha, \beta)= & \prod_{i=1}^{n} f_{N F W}\left(t_{i} ; \mu, \alpha, \beta\right) \\
= & \prod_{i=1}^{n} \exp (\mu)\left\{\exp \left(t_{i}\right)-1\right\}^{\alpha-1}\left\{\log \left(t_{i}+1\right)\right\}^{\beta-1} \\
& \times\left[\alpha \exp \left(t_{i}\right) \log \left(t_{i}+1\right)+\left\{\exp \left(t_{i}\right)-1\right\} \frac{\beta}{t_{i}+1}\right] \\
& \times \exp \left[-\exp (\mu)\left\{\exp \left(t_{i}\right)-1\right\}^{\alpha}\left\{\log \left(t_{i}+1\right)\right\}^{\beta}\right] .
\end{aligned}
$$

Then the score functions for parameters $\mu, \alpha$ and $\beta$ can be obtained as follows.

$$
\begin{aligned}
\frac{\partial \log L_{N F W}}{\partial \mu}= & n-\sum_{i=1}^{n} \exp (\mu)\left\{\exp \left(t_{i}\right)-1\right\}^{\alpha}\left\{\log \left(t_{i}+1\right)\right\}^{\beta}, \\
\frac{\partial \log L_{N F W}}{\partial \alpha}= & \sum_{i=1}^{n} \log \left\{\exp \left(t_{i}\right)-1\right\}+\sum_{i=1}^{n} \frac{\exp \left(t_{i}\right) \log \left(t_{i}+1\right)\left(t_{i}+1\right)}{\alpha \exp \left(t_{i}\right) \log \left(t_{i}+1\right)\left(t_{i}+1\right)+\beta\left\{\exp \left(t_{i}\right)-1\right\}} \\
& -\exp (\mu) \sum_{i=1}^{n}\left\{\exp \left(t_{i}\right)-1\right\}^{\alpha}\left\{\log \left(t_{i}+1\right)\right\}^{\beta} \log \left\{\exp \left(t_{i}\right)-1\right\}, \\
\frac{\partial \log L_{N F W}}{\partial \beta}= & \sum_{i=1}^{n} \log \left\{\log \left(t_{i}+1\right)\right\}+\sum_{i=1}^{n} \frac{\left\{\exp \left(t_{i}\right)-1\right\}}{\alpha \exp \left(t_{i}\right) \log \left(t_{i}+1\right)\left(t_{i}+1\right)+\beta\left\{\exp \left(t_{i}\right)-1\right\}} \\
& -\exp (\mu) \sum_{i=1}^{n}\left\{\exp \left(t_{i}\right)-1\right\}^{\alpha}\left\{\log \left(t_{i}+1\right)\right\}^{\beta} \log \left\{\log \left(t_{i}+1\right)\right\} .
\end{aligned}
$$

In order to obtain maximum likelihood estimates of $\mu, \alpha$ and $\beta$, we solve the above three equations using the quasi-Newton method with initial values set to the ordinary least square estimates.

\section{Simulation studies}

In order to evaluate the performance of the new flexible Weibull distribution, we consider the following five different types of distributions. The hazard functions are given in Figure 7.

Example 1. Constant hazard function: Exponential distribution with a rate parameter $\lambda=1 / 3$.

Example 2. Decreasing hazard function: Additive Weibull distribution suggested by Lemonte et al. (2014) with parameters $a=0.1, b=1, c=0.2, d=0.8$.

Example 3. Increasing hazard function: Additive Weibull distribution suggested by Lemonte et al. (2014) with parameters $a=0.1, b=1.5, c=0.2, d=1$. 

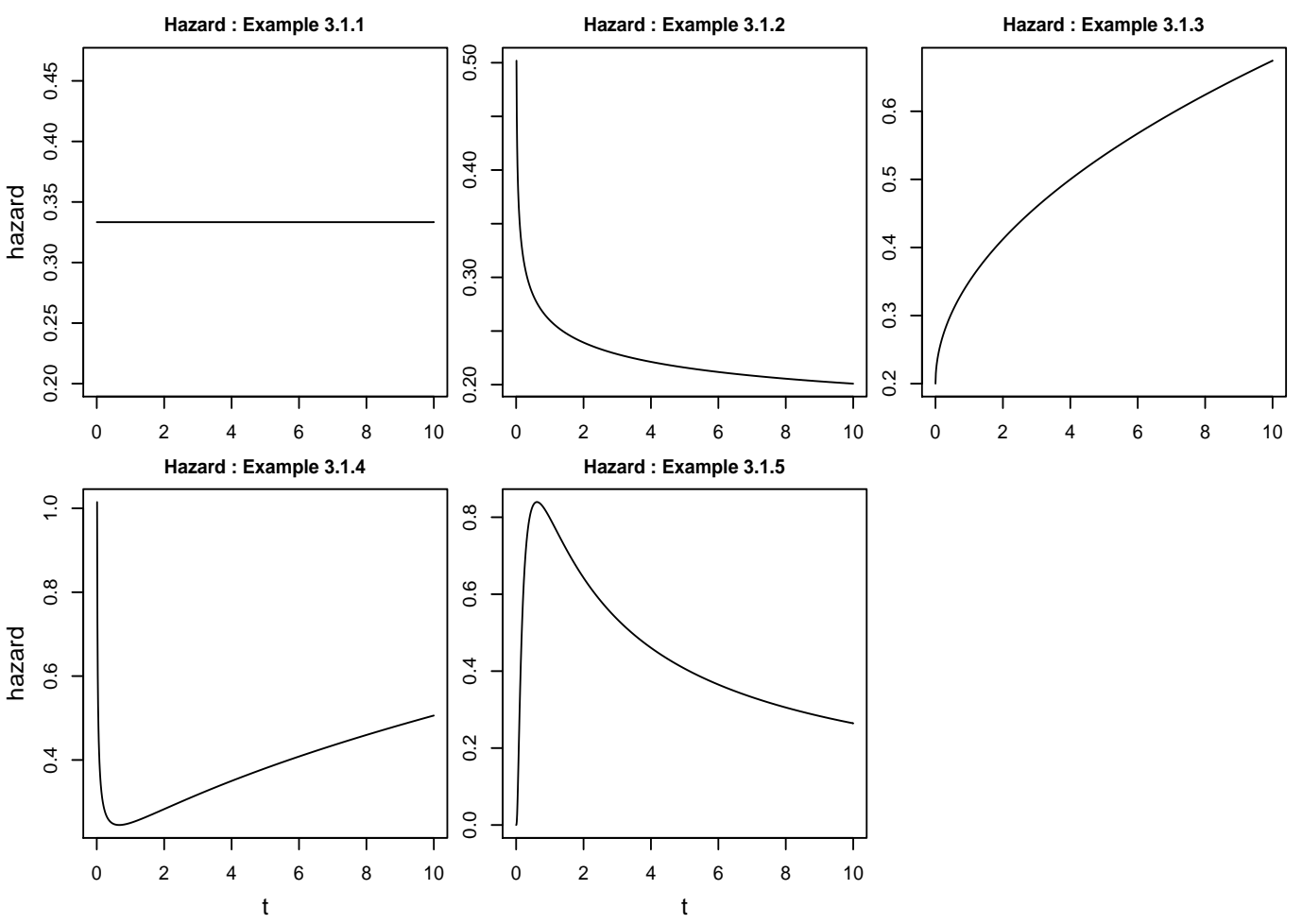

Figure 7: Hazard functions for simulated examples.

Example 4. Bathtub-shaped hazard function: Additive Weibull distribution suggested by Lemonte et al. (2014) with parameters $a=0.1, b=1.5, c=0.2, d=0.5$.

Example 5. Upside-down bathtub-shaped hazard function: Lognormal distribution with mean $\mu=$ 0 and variance $\sigma=1$.

We compare the new flexible Weibull distribution with the standard Weibull distribution (twoparameter), modified Weibull distribution (three- parameter), flexible Weibull distribution (threeparameter), very flexible Weibull distribution (three-parameter), and two-parameter lifetime distribution suggested by Chen (2000) as

1. Weibull

$$
\log H(t)=\alpha+\beta \log (t)
$$

2. Modified Weibull by Lai et al. (2003)

$$
\log H(t)=\mu+\alpha t+\beta \log (t) .
$$

3. Flexible Weibull by Bebbington et al. (2007)

$$
\log H(t)=\alpha t-\frac{\beta}{t}
$$


Table 1: Maximum likelihood fit of Examples 1-5

\begin{tabular}{|c|c|c|c|c|c|c|}
\hline \multirow{2}{*}{ Model } & \multicolumn{3}{|c|}{ Example 1} & \multicolumn{3}{|c|}{ Example 2} \\
\hline & $\log L$ & AIC & $\mathrm{K}-\mathrm{S}$ & $\log L$ & AIC & $\mathrm{K}-\mathrm{S}$ \\
\hline Weibull & -40.8832 & 85.7664 & 0.1323 & -47.2960 & 98.5919 & 0.1325 \\
\hline Modified Weibull & -40.7016 & 87.4032 & 0.1266 & -46.9051 & 99.8103 & 0.1258 \\
\hline Flexible Weibull & -43.8693 & 91.7386 & 0.2816 & -51.3680 & 106.7360 & 0.3132 \\
\hline Very flexible Weibull & -40.2433 & 86.4866 & 0.1215 & -46.6124 & 99.2249 & 0.1217 \\
\hline Chen & -41.5697 & 87.1394 & 0.1419 & -47.9022 & 99.8043 & 0.1406 \\
\hline New flexible Weibull & -40.2352 & 86.4703 & 0.1217 & -46.6118 & 99.2237 & 0.1219 \\
\hline \multirow{2}{*}{ Model } & \multicolumn{3}{|c|}{ Example 3} & \multicolumn{3}{|c|}{ Example 4} \\
\hline & $\log L$ & AIC & $\mathrm{K}-\mathrm{S}$ & $\log L$ & AIC & $\mathrm{K}-\mathrm{S}$ \\
\hline Weibull & -36.9378 & 77.8755 & 0.1328 & -41.3283 & 86.6566 & 0.1476 \\
\hline Modified Weibull & -36.5120 & 79.0239 & 0.1247 & -39.8640 & 85.7279 & 0.1219 \\
\hline Flexible Weibull & -39.5647 & 83.1295 & 0.2703 & -46.8934 & 97.7868 & 0.4069 \\
\hline Very flexible Weibull & -36.2870 & 78.5740 & 0.1212 & -39.8316 & 85.6633 & 0.1228 \\
\hline Chen & -37.3808 & 78.7615 & 0.1385 & -40.4268 & 84.8537 & 0.1300 \\
\hline New flexible Weibull & -36.2783 & 78.5566 & 0.1212 & -39.8612 & 85.7224 & 0.1235 \\
\hline \multirow{2}{*}{ Model } & \multicolumn{3}{|c|}{ Example 5} & & & \\
\hline & $\log L$ & AIC & $\mathrm{K}-\mathrm{S}$ & & & \\
\hline Weibull & -43.3817 & 90.7635 & 0.1319 & & & \\
\hline Modified Weibull & -43.3713 & 92.7427 & 0.1318 & & & \\
\hline Flexible Weibull & -42.7599 & 89.5197 & 0.1691 & & & \\
\hline Very flexible Weibull & -41.7097 & 89.4194 & 0.1100 & & & \\
\hline Chen & -46.8947 & 97.7893 & 0.1681 & & & \\
\hline New flexible Weibull & -41.7001 & 89.4003 & 0.1101 & & & \\
\hline
\end{tabular}

4. Very flexible Weibull by Park and Park (2016)

$$
\log H(t)=\mu+\alpha t+\beta \log \log (t+1) .
$$

5. Two-parameter lifetime distribution by Chen (2000)

$$
\log H(t)=\mu+\log \left(\exp \left(t^{\beta}\right)-1\right)
$$

As we can see, the above four modified Weibull distributions contain at least one inappropriate cumulative hazard function.

In evaluating the performance, we generated a random sample of size 30 from each distribution, and calculated the log-likelihood value, Akaike Information Criterion (AIC), and KolmogorovSmirnov statistic (K-S). We repeated 100,000 Monte Carlo simulations and calculated the averages which are tabulated in Table 1.

The numerical results in Table 1 indicate that the new flexible Weibull distribution shows the robust performances over the five different distributions. For the first three examples where the Weibull distribution can fit well, the Weibull distribution shows the lowest AIC values but the new flexible Weibull shows the second lowest AIC values. For the fourth and fifth examples where the Weibull distribution can not fit well, the new flexible Weibull distribution shows the second lowest AIC value for the fourth case and lowest AIC value for the fifth case.

\section{Application}

We use a real data example to illustrate the efficiency of new flexible Weibull distribution. The failure time data studied in Murthy et al. (2004) and Aryal and Elbatal (2015) were used for application. The 

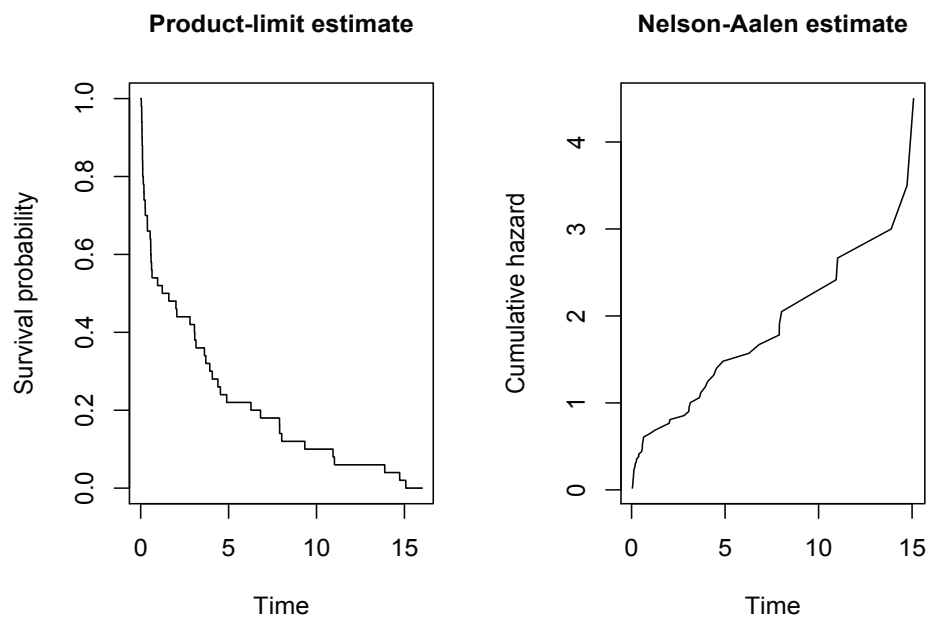

Figure 8: Survival function and cumulative hazard function for real data example.

Table 2: Maximum likelihood fit of Example 6

\begin{tabular}{cccc}
\hline \hline Model & $\log L$ & AIC & K-S \\
\hline Weibull & -102.3643 & 208.7286 & 0.1270 \\
Modified Weibull & -101.3636 & 208.7273 & 0.1326 \\
Flexible Weibull & -95.9229 & 195.8457 & 0.1878 \\
Very flexible Weibull & -99.4470 & 204.8941 & 0.1123 \\
Chen et al. & -103.4204 & 210.8408 & 0.1397 \\
New flexible Weibull & -99.3345 & 204.6691 & 0.1103 \\
\hline
\end{tabular}

AIC = Akaike Information Criterion; K-S = Kolmogorov-Smirnov.

data are as follows.

Example 6. 0.036, 0.058, 0.061, 0.074, 0.078, 0.086, 0.102, 0.103, 0.114, 0.116, 0.148, 0.183, $0.192,0.254,0.262,0.379,0.381,0.538,0.570,0.574,0.590,0.618,0.645,0.961,1.228,1.600$, $2.006,2.054,2.804,3.058,3.076,3.147,3.625,3.704,3.931,4.073,4.393,4.534,4.893,6.274$, $6.816,7.896,7.904,8.022,9.337,10.940,11.020,13.880,14.730,15.080$.

Figure 8 illustrates the survival function and cumulative hazard function using product-limit estimate and Nelson-Aalen estimate, respectively. We compare the efficiency of new flexible Weibull distribution with aforementioned distributions using log-likelihood value, AIC and K-S values (Table 2).

The results in Table 2 indicate that flexible Weibull distribution shows the largest log-likelihood value and lowest AIC value; however, flexible Weibull distribution has the poorest fit in terms of $\mathrm{K}-\mathrm{S}$ value. On the other hand, new flexible Weibull distribution shows second best fit in terms of log-likelihood value and AIC value along with lowest K-S value.

\section{Conclusions}

Some well-known modified Weibull distributions can be represented as a multiplication of two cumulative hazard functions, but some cumulative hazard functions do not satisfy the properties as a cumulative hazard function. We consider $\exp (t)-1$ and $\log (t+1)$, and suggest a new modified Weibull 
distribution called a new flexible Weibull distribution which is theoretically rigorous and shows more flexibility. The hazard function of the new flexible Weibull distribution can cover the monotone shape as well as non-monotone shape that include bathtub-shaped, modified bathtub-shaped or upside-down bathtub-shaped. We presented the parameter estimation methods and compared their performance with some modified Weibull distributions for various types of distributions and real data application.

\section{Acknowledgement}

This research was supported by Basic Science Research Program through the National Research Foundation of Korea (NRF) funded by the Ministry of Education (NRF-2015R1A2A1A15051493).

\section{References}

Almalki SJ and Yuan J (2013). A new modified Weibull distribution, Reliability Engineering and System Safety, 111, 164-170.

Aryal G and Elbatal I (2015). On the exponentiated generalized modified Weibull distribution, Communications for Statistical Applications and Methods, 22, 333-348.

Bebbington M, Lai CD, and Zitikis R (2007). A flexible Weibull extension, Reliability Engineering and System Safety, 92, 719-726.

Chen Z (2000). A new two-parameter lifetime distribution with bathtub shape or increasing failure rate function, Statistics and Probability Letters, 49, 155-161.

Lai CD, Xie M, and Murthy DNP (2003). A modified Weibull distribution, IEEE Transactions on Reliability, 52, 33-37.

Lemonte AJ, Cordeiro GM, and Ortega EM (2014). On the additive Weibull distribution, Communications in Statistics-Theory and Methods, 43, 2066-2080.

Murthy DNP, Xie M, and Jiang R (2004). Weibull Models, John Wiley \& Sons Inc., Hoboken, NJ.

Nadarajah S and Kotz S (2005). On some recent modification of Weibull distributions, IEEE Transactions on Reliability, 54, 561-562.

Park S and Park J (2016). A general class of flexible Weibull distributions, Communications in Statistics - Theory and Methods. Available from: http://dx.doi.org/10.1080/03610926.2015.1118509

Xie M and Lai CD (1995). Reliability analysis using an additive Weibull model with bathtub-shaped failure rate function, Reliability Engineering and System Safety, 52, 87-93.

Xie M, Tang Y, and Goh TN (2002). A modified Weibull extension with bathtub-shaped failure rate function, Reliability Engineering and System Safety, 76, 279-285. 